\title{
The role of unintended pregnancy in internalized stigma among women living with HIV in Kenya
}

\author{
Sara Chace Dwyer ${ }^{1 *+}$, Aparna Jain ${ }^{1 \dagger}$, Wilson Liambila ${ }^{2}$ and Charlotte E. Warren ${ }^{1}$
}

\begin{abstract}
Background: Kenya has successfully expanded HIV treatment, but HIV-related stigma and discrimination, and unintended pregnancy remain issues for many Kenyan women living with HIV. While HIV-related stigma can influence the health seeking behaviors of those living with HIV, less is known about how reproductive health outcomes influence internalized stigma among women living with HIV.

Methods: Baseline data only were used in this analysis and came from an implementation science study conducted in Kenya from 2015 to 2017. The analytic sample was limited to 1116 women who are living with HIV, between 18 to 44 years old, and have ever experienced a pregnancy. The outcome variable was constructed from 7 internalized stigma statements and agreement with at least 3 statements was categorized as medium/high levels of internalized stigma. Unintended pregnancy, categorized as unintended if the last pregnancy was mistimed or unwanted, was the key independent variable. Univariate and multivariate logistic regression models were used to assess the association between unintended pregnancy and internalized stigma. Associations between internalized stigma and HIV-related discrimination and violence/abuse were also explored.
\end{abstract}

Results: About 48\% agreed with at least one internalized stigma statement and 19\% agreed with at least three. Over half of women reported that their last pregnancy was unintended (59\%). Within the year preceding the survey, $52 \%$ reported experiencing discrimination and $41 \%$ reported experiencing violence or abuse due to their HIV status. Women whose last pregnancy was unintended were 1.6 times (95\% Cl 1.2-2.3) more likely to have medium/high levels of internalized stigma compared to those whose pregnancy was wanted at the time, adjusting for respondents' characteristics, experiences of discrimination, and experiences of violence and abuse. Women who experienced HIVrelated discrimination in the past 12 months were 1.8 times (95\% Cl 1.3-2.6) more likely to have medium/high levels of internalized stigma compared to those who experienced no discrimination.

Conclusions: Results suggest that unintended pregnancy is associated with internalized stigma. Integrated HIV and FP programs in Kenya should continue to address stigma and discrimination while increasing access to comprehensive voluntary family planning services for women living with HIV.

\footnotetext{
*Correspondence: schace@popcouncil.org

${ }^{\dagger}$ Sara A. Chace Dwyer and Aparna Jain have contributed equally to the work

1 Population Council, 4301 Connecticut Ave NW \# 280, Washington, DC 20008, USA

Full list of author information is available at the end of the article
} original author(s) and the source, provide a link to the Creative Commons licence, and indicate if changes were made. The images or other third party material in this article are included in the article's Creative Commons licence, unless indicated otherwise in a credit line to the material. If material is not included in the article's Creative Commons licence and your intended use is not permitted by statutory regulation or exceeds the permitted use, you will need to obtain permission directly from the copyright holder. To view a copy of this licence, visit http://creativecommons.org/licenses/by/4.0/. The Creative Commons Public Domain Dedication waiver (http://creativeco mmons.org/publicdomain/zero/1.0/) applies to the data made available in this article, unless otherwise stated in a credit line to the data. 
Keywords: Internalized stigma, Family planning, Unintended pregnancy, Reproductive health, HIV-related stigma and discrimination

\section{Background}

In Kenya, it is estimated that 1.6 million people (approximately $3 \%$ of Kenya's population) are living with HIV as of 2018 and women ages 15 years and older account for about $57 \%$ of all individuals living with HIV [1]. This is slightly higher than the global average, where women account for $50 \%$ of those living with HIV [2]. The HIV prevalence rate for women of reproductive age (1549 years) in Kenya is almost double the rate for men of the same age (6.1 compared to 3.4, respectively) [1].

Kenya has successfully expanded access to HIV services in the last decade. Among Kenyan women living with HIV (WLHIV), about $75 \%$ are on antiretroviral therapy (ART) in 2018 compared to 29\% in 2010 [1]. Most pregnant WLHIV (91\%) in Kenya receive antiretroviral drugs as part of prevention of mother-to-child transmission strategies [1], which has been proven to significantly reduce the risk of transmission [3]. Prevention of mother-to-child transmission strategies have led to an estimated 1.4 child infections averted [4] making it possible for women and couples living with HIV to have children without transmitting HIV during pregnancy and the postpartum period [5]. Yet WLHIV who wish to avoid pregnancy also need comprehensive family planning (FP) services [6]. Studies in Kenya have shown that WLHIV want to avoid pregnancy more so compared to HIVnegative women [6-8], consistent with other studies conducted in Malawi [7, 9], sub-Saharan Africa broadly [10], and the United States [11]. Women's fertility decisions have been found to change within one week of receiving a HIV-positive diagnosis [9] and reasons for wanting fewer children include concerns over transmitting HIV to their child, not being able to care for their children, greater risk to the mother's health, and increased financial burden $[7,8,11]$. Some studies have found that individuals living with HIV may decide to have additional children as they begin to feel healthier with ARTs [10, 12].

While many HIV-positive women may want to avoid a pregnancy, unintended pregnancy (the percentage of pregnancies that were wanted later or not wanted at all) is generally high among WLHIV. In Kenya, unintended pregnancy among WLHIV was found to be between 43 and $59 \%[6,8,13]$, which is higher than the national estimates for all women age 15-49 (36\%) [14]. Studies conducted in other countries have also found high levels of unintended pregnancy among WLHIV: between 68 and $71 \%$ in South Africa [15-17]; 66-92\% across sub-Saharan Africa [10]; 60\% in Canada [18]; and $83 \%$ in the United
States [19]. Evidence of differences in unintended pregnancy comparing WLHIV and HIV-negative women is mixed. Studies in Kenya [6], Ethiopia [20] and South Africa [21] found WLHIV reported higher rates of unintended pregnancy compared to HIV-negative women. A study conducted in Nigeria and Zambia, however, found no difference in the odds of unintended pregnancy by HIV status [22]. The evidence around contraceptive use among WLHIV compared HIV-negative Kenyan women is also mixed $[6,7,23]$.

\section{HIV-related stigma and discrimination}

HIV-related stigma and discrimination involve negative attitudes and behaviors anyone can have, including family, friends, health care workers, and community members, towards people living with HIV [24]. People living with HIV can also experience internalized stigma, by having negative feelings and thoughts about themselves because of their HIV status [25], and anticipated stigma, which is the belief that they will experience stigma, discrimination or abuse from others because of their HIV status [26]. HIV-related stigma and anticipated stigma can influence the health seeking behaviors of people living with HIV including ART adherence [16, 27-32]. A systematic review conducted in 2013 found mixed results on the effects of internalized stigma on ART adherence [28] but more recent studies have documented an association between internalized stigma and low ART adherence [32-34] and low ART initiation [35]. In Kenya, anticipated stigma was found to influence HIV testing among pregnant women $[26,31]$ while HIV-related stigma and discrimination were reasons why pregnant WLHIV reported avoiding delivery in a health facility [29]. Internalized stigma can also influence a person's wellbeing and has been linked to depression and thoughts of suicide. For instance, postpartum women in Kenya who experienced shame over their HIV status were more depressed compared to postpartum women who reported social rejection [36]. Qualitative studies in Kenya have also shown that some WLHIV prefer to receive FP services from facilities specializing in HIV services [30].

The effects of HIV-related stigma and discrimination on women's wellbeing and health-seeking behaviors have been well documented. Little is known, however, about the association between reproductive health outcomes and internalized stigma experienced by WLHIV. This paper aims to contribute to the literature by 
examining the relationship between unintended pregnancy and internalized stigma among HIV-positive women in south western Kenya.

\section{Study locations}

The study was conducted in Samia and Butula subcounties of Busia County, which are located in south western Kenya. Samia has a population of 107,176 and Butula has a population of 140,334 [37]. Both sub-counties have similar socioeconomic characteristics [38]. The HIV prevalence in Busia county is $10 \%$, double the national average (5\%) for adults aged 15-64 years [39]. The estimated HIV prevalence rate among women is $9 \%$ in Busia county compared to 5\% nationally [40].

\section{Methods}

\section{Data source}

The baseline data used in this analysis were part of larger implementation science study that tested a community-based approach for integrated FP and HIV services between 2015 and 2017. Quantitative interviews were conducted with WLHIV before and after the community-based FP/HIV intervention was implemented [38]. The intervention did not look at reducing HIVrelated stigma. The study protocol received IRB approvals from the Population Council (Protocol 702) and the Kenyatta National Hospital/University of Nairobi Ethics Research Committee (Protocol P573/08/2015).

Women between the ages of 18-49 were enrolled through HIV support groups. The support groups provided their members with information about the study and women who were interested in participating were contacted by research assistants trained in research ethics, implementing informed consent forms, and the study's design, objectives, and questionnaires. The research assistants then met with potential respondents, provided them with the purpose and objectives of the study and obtained informed consent. Research assistants used tablets to administer a quantitative questionnaire. The questionnaire collected data on socio-demographic characteristics, general health status, health-seeking behaviors, FP use, use of HIV services, and perceived or experienced stigma and discrimination (Additional file 1). Interviews were conducted in Kiswahili and responses in the tablet were recorded in English. A total of 1609 women participated in the implementation science study: 1090 in Samia and 519 in Butula.

\section{Dependent variable}

The dependent variable is internalized stigma, categorized as medium/high versus low. The internalized stigma variable was created from seven questions that were adapted from the HIV Stigma Scale [41]. Respondents were asked to answer a series of attitudinal questions on a 4-point Likert scale ranging from strongly disagree to strongly agree. Responses for each item were then dichotomized into strongly agree/agree or strongly disagree/disagree. The seven items were combined to form an additive index ranging from 0 to 7 , where higher values represented higher levels of internalized stigma. The internalized stigma variable was dichotomized into low and medium/high internalized stigma. Low internalized stigma included women who agreed with 0 to 2 items (coded as 0 ) and medium/ high internalized stigma included those who agreed with 3 or more items (coded as 1).

\section{Independent variables Unintended pregnancy}

Women were asked "At the time you became pregnant with your last child, did you intend to become pregnant then, did you want to wait until later, or did you not want to have any (more) children?". A dichotomized variable was created where unintended pregnancy included women who reported that their last pregnancy was wanted later or not at all (coded as 1) and intended pregnancy included women whose last pregnancy was wanted at the time they became pregnant (coded as 0 ).

\section{Experience of HIV-related discrimination and violence orabuse}

Two variables, experience of HIV related discrimination and experience of violence or abuse, were used to adjust the model because of their known relationship to internalized stigma. Women were asked about their experiences with discrimination related to their HIV status. They were asked four questions about discrimination that occurred in the past 12 months: excluded from family activities; aware of being gossiped about; rejected by a sexual partner; and treated unfairly or discriminated against by a healthcare provider. Women who did not experience discrimination related to any of the four questions were coded as 0 , and women who experienced 1 or more of the questions were coded as 1-

Experience of violence or abuse was measured by three questions. Women were asked if they were verbally insulted, physically abused, and sexually assaulted or forced to have sex in the past 12 months. A dichotomized variable was constructed where 1 was coded if 
the respondent had answered yes to at least one questions and 0 if she did not experience violence on all 3 questions.

\section{Additional variables}

Additional covariates included age, marital status, number of living children, having one or more children living with HIV, education, length of time living in same village, self-reported health status, length of time using antiretrovirals (ARVs) and sub-county residence.

\section{Data analysis}

The analytic sample was limited to respondents who were 18 to 44 years of age and had ever been pregnant or were currently pregnant $(n=1116)$ at the time of the survey. Descriptive statistics were calculated for respondent characteristics, unintended last pregnancy, HIV-related discrimination, experience of violence, and reported experience of internalized stigma. Univariate and multivariate logistic regression models were used to assess the association between unintended pregnancy and internalized stigma. The analysis were conducted in STATA.SE, Version 13.

\section{Results}

Table 1 shows the demographic profile of respondents living with HIV, and experience of internalized stigma, HIV-related discrimination, and violence or abuse. Over half $(56 \%)$ of women were in the latter half of their reproductive life (30\% were aged $35-39$ and $26 \%$ were aged 40-44). Many (63\%) were married at the time of the interview and $42 \%$ had between 3-4 living children. Few women $(13 \%)$ reported that one or more of their children were HIV positive. Most women did not complete primary school (78\%), and 68\% lived in the same village for over 10 years. More women were from Samia sub-county (70\%). The majority rated their health as fair or good (90\%) and 78\% were using ARVs for over a 1 year.

More than half $(59 \%)$ of women reported that their last pregnancy was unintended (Table 1). Among those who reported that their last pregnancy was unintended $(n=654), 57 \%$ were not using contraception at the time and the most commonly cited reasons for not using FP among these women included: didn't know about FP or where to get it/lack of access (14\%); partner didn't want them to use FP or others advised them against using FP (14\%); fear of side effects/because contraceptive methods interfered with their bodily processes (12\%); and not wanting to use FP (10\%) (data not shown). Nineteen percent of women experienced medium/high level of internalized stigma (Table 1 ).
Table 1 Study sample characteristics $(n=1116)$

\begin{tabular}{|c|c|}
\hline & $\%$ \\
\hline \multicolumn{2}{|l|}{ Age } \\
\hline $18-24$ & 6.7 \\
\hline $25-29$ & 16.6 \\
\hline $30-34$ & 20.8 \\
\hline $35-39$ & 29.9 \\
\hline $40-44$ & 26.0 \\
\hline \multicolumn{2}{|l|}{ Marital status } \\
\hline Single & 2.6 \\
\hline Living together & 2.7 \\
\hline Married & 62.8 \\
\hline Divorced/separated/widowed & 31.9 \\
\hline \multicolumn{2}{|l|}{ Number of living children } \\
\hline 0 & 2.5 \\
\hline $1-2$ & 23.8 \\
\hline $3-4$ & 41.6 \\
\hline $5+$ & 32.1 \\
\hline \multicolumn{2}{|l|}{ Number of children living with HIV } \\
\hline At least 1 & 12.5 \\
\hline None/don't know & 87.5 \\
\hline \multicolumn{2}{|l|}{ Education } \\
\hline None & 9.9 \\
\hline Did not complete primary & 78.3 \\
\hline Completed primary & 10.8 \\
\hline Secondary or more & 1.0 \\
\hline \multicolumn{2}{|l|}{ Duration living in same village } \\
\hline$<10$ years & 32.0 \\
\hline$\geq 10$ years & 68.0 \\
\hline \multicolumn{2}{|l|}{ Sub-County } \\
\hline Samia & 70.3 \\
\hline Butula & 29.7 \\
\hline \multicolumn{2}{|l|}{ Self-reported health status } \\
\hline Poor/Don't know & 9.5 \\
\hline Fair & 41.3 \\
\hline Good & 49.2 \\
\hline \multicolumn{2}{|l|}{ Length of time on ARV treatment } \\
\hline No treatment/1 year or less & 21.9 \\
\hline More than 1 year & 78.1 \\
\hline \multicolumn{2}{|l|}{ Unintended last pregnancy } \\
\hline Yes (wanted pregnancy later/did not want pregnancy at all) & 58.6 \\
\hline No (wanted pregnancy then) & 41.4 \\
\hline \multicolumn{2}{|l|}{ Internalized stigma } \\
\hline Medium/high & 19.1 \\
\hline Low/none & 80.9 \\
\hline
\end{tabular}

Figure 1 shows the percent of respondents who experienced HIV-related discrimination in the 12 months preceding the interview. Just over half of respondents did not experience any HIV related discrimination. Just under half said that they were gossiped about because of their 


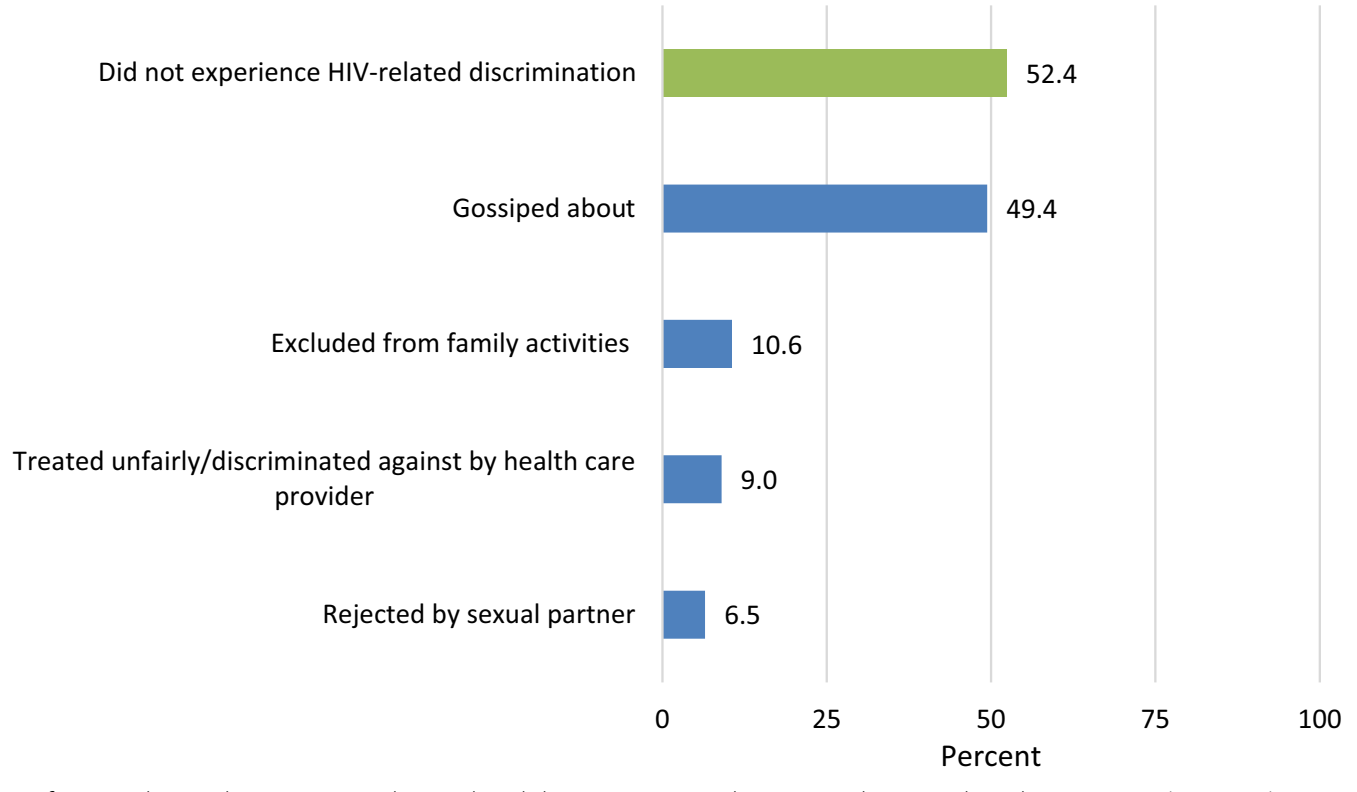

Fig. 1 Percent of respondents who experienced HIV-related discrimination in the 12 months preceding the interview $(n=1116)$

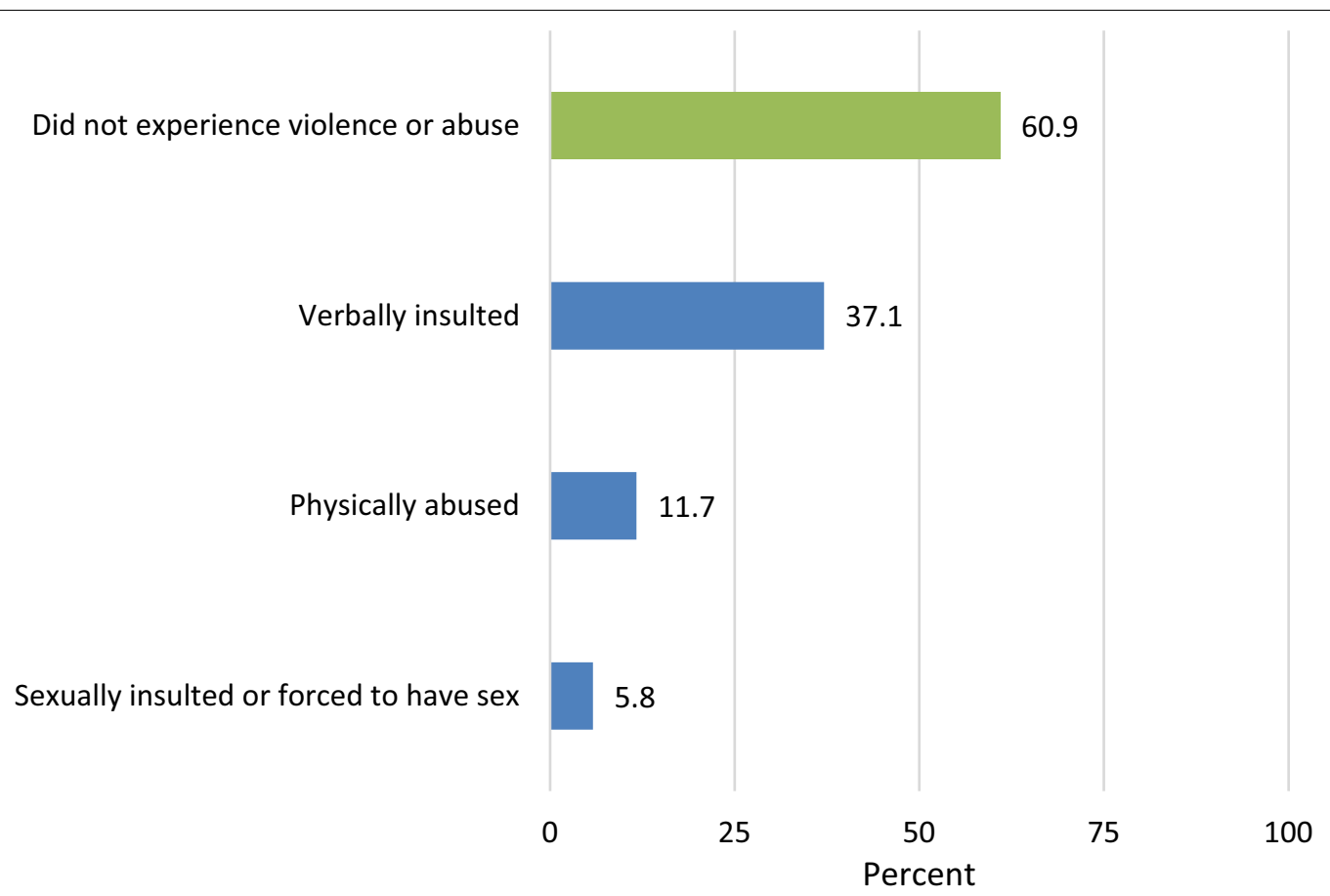

Fig. 2 Percent of respondents who experienced violence or abuse in the 12 months preceding the interview $(n=1116)$

HIV status and $11 \%$ were excluded from family activities. Few said that they were treated unfairly by a health care provider $(9 \%)$ or were rejected by a sexual partner (7\%).

Figure 2 shows the percent of respondents who experienced violence within the 12 months preceding the interview. Thirty-seven percent had been verbally insulted and $12 \%$ had been physically abused. Six percent had been sexually insulted or forced to have sex within the past 12 months. 
Did not agree with any internalized stigma statement

People's attitudes towards PLWHIV make me feel worse about myself

Having HIV in my body is disgusting to me

I feel guilty because I have HIV

I feel ashamed because of having HIV

I feel I'm not as good as others because I have HIV

Having HIV makes me feel I'm a bad person

Having HIV makes me feel unclean

0

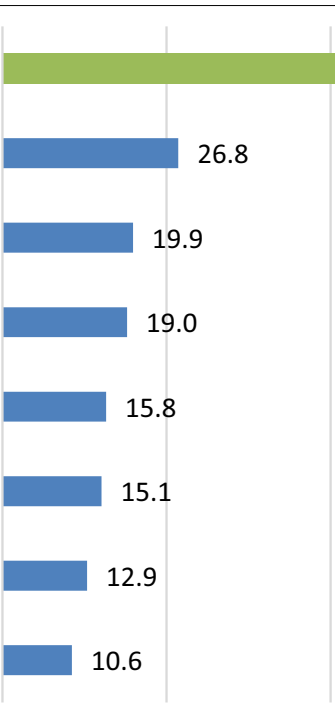

25
52.6

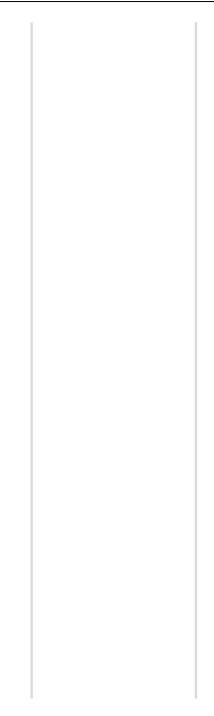

75

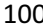

Fig. 3 Percent of respondents who agreed with internalized stigma statements $(n=1116)$

75

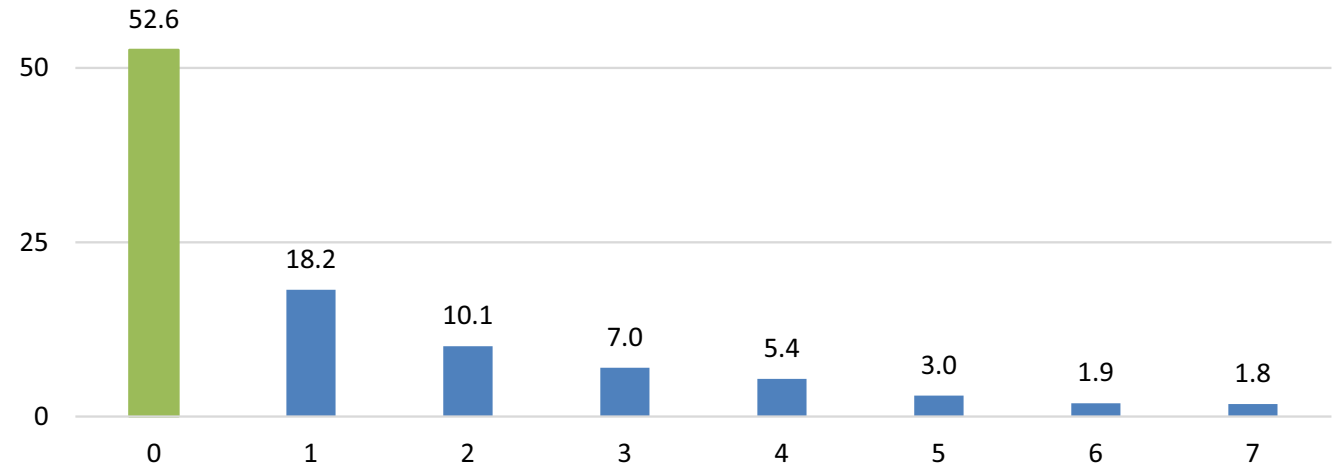

Fig. 4 Distribution of respondents by number of internalized stigma statements agreed to $(n=116)$

\section{Internalized stigma}

Figure 3 shows women's responses to seven internalized stigma statements. Over a quarter (27\%) of women agreed with the statement "people's attitudes towards people living with HIV makes me feel worse about myself." Twenty percent agreed with the statement "having HIV in body is disgusting to me" and 19\% agreed with
"I feel guilty because I have HIV." Between 11 and 16\% agreed with the other four internalized stigma measures. Figure 4 shows the percent of respondents who agreed with multiple internalized stigma statements. More than half (53\%) did not agree with any internalized stigma statement, $18 \%$ agreed with 1 statement, $10 \%$ 
Table 2 Unadjusted and adjusted logistic regression models of unintended last pregnancy on medium to high internalized stigma among women who have ever been pregnant $(n=1116)$

\begin{tabular}{|c|c|c|c|c|}
\hline & \multicolumn{2}{|c|}{ Unadjusted Model } & \multicolumn{2}{|c|}{ Adjusted model } \\
\hline & OR & $95 \% \mathrm{Cl}$ & $\mathrm{AOR}$ & $95 \% \mathrm{Cl}$ \\
\hline \multicolumn{5}{|l|}{ Unintended last pregnancy } \\
\hline Yes & $1.74^{* * *}$ & $(1.26-2.39)$ & $1.64^{* *}$ & $(1.16-2.30)$ \\
\hline No & Ref & & Ref & \\
\hline \multicolumn{5}{|c|}{ Experienced HIV-related discrimination } \\
\hline Yes & $2.02^{* * *}$ & $(1.48-2.77)$ & $1.79^{* * *}$ & $(1.25-2.58)$ \\
\hline No & Ref & & Ref & \\
\hline \multicolumn{5}{|c|}{ Experienced HIV-related violence/abuse } \\
\hline Yes & $1.63^{* * *}$ & $(1.21-2.20)$ & 1.17 & $(0.82-1.66)$ \\
\hline No & Ref & & Ref & \\
\hline \multicolumn{5}{|c|}{ Length of time on ARV treatment } \\
\hline No treatment/ 1 year or less & 0.83 & $(0.58-1.18)$ & 0.91 & $(0.62-1.33)$ \\
\hline More than 1 year & Ref & & Ref & \\
\hline \multicolumn{5}{|l|}{ Age } \\
\hline $18-24$ & 1.13 & $(0.62-2.06)$ & 0.96 & $(0.49-1.90)$ \\
\hline $25-29$ & 0.77 & $(0.48-1.23)$ & 0.78 & $(0.47-1.29)$ \\
\hline $30-34$ & 0.73 & $(0.47-1.13)$ & 0.72 & $(0.46-1.15)$ \\
\hline $35-39$ & Ref & & Ref & \\
\hline $40-44$ & 0.98 & $(0.66-1.45)$ & 0.94 & $(0.62-1.41)$ \\
\hline \multicolumn{5}{|l|}{ Marital status } \\
\hline Single & 1.28 & $(0.51-3.20)$ & 1.06 & $(0.40-2.81)$ \\
\hline Living together & 2.10 & $(0.94-4.69)$ & 1.76 & $(0.76-4.04)$ \\
\hline Married & Ref & & Ref & \\
\hline $\begin{array}{l}\text { Divorced/separated/wid- } \\
\text { owed }\end{array}$ & $1.39^{*}$ & $(1.01-1.92)$ & 1.31 & $(0.93-1.85)$ \\
\hline \multicolumn{5}{|l|}{ Number of living children } \\
\hline 0 & 1.71 & $(0.73-4.01)$ & 2.00 & $(0.81-4.93)$ \\
\hline $1-2$ & 1.06 & $(0.73-1.55)$ & 1.04 & $(0.69-1.58)$ \\
\hline $3-4$ & Ref & & Ref & \\
\hline $5+$ & 0.93 & $(0.65-1.33)$ & 0.80 & $(0.54-1.18)$ \\
\hline \multicolumn{5}{|l|}{ Children living with HIV } \\
\hline At least 1 & 0.81 & $(0.51-1.31)$ & 0.74 & $(0.45-1.22)$ \\
\hline None/don't know & Ref & & Ref & \\
\hline \multicolumn{5}{|l|}{ Education } \\
\hline None & 1.14 & $(0.70-1.85)$ & 1.09 & $(0.65-1.81)$ \\
\hline Did not complete primary & Ref & & Ref & \\
\hline Completed primary & 0.86 & $(0.52-1.43)$ & 0.81 & $(0.48-1.38)$ \\
\hline Secondary or more & 3.07 & $(0.96-9.79)$ & 2.91 & $(0.84-10.16)$ \\
\hline \multicolumn{5}{|l|}{ Duration living in same village } \\
\hline$<10$ years & 0.92 & $(0.67-1.27)$ & 0.97 & $(0.68-1.39)$ \\
\hline$\geq 10$ years & Ref & & Ref & \\
\hline \multicolumn{5}{|l|}{ Sub-County } \\
\hline Butula & Ref & & Ref & \\
\hline Samia & $1.92^{* * *}$ & $(1.33-2.77)$ & $1.90^{* * *}$ & $(1.30-2.77)$ \\
\hline \multicolumn{5}{|l|}{ Self-reported health status } \\
\hline Poor/don't know & $1.65^{*}$ & $(1.02-2.66)$ & 1.44 & $(0.87-2.38)$ \\
\hline Fair & 0.95 & $(0.69-1.31)$ & 0.87 & $(0.62-1.21)$ \\
\hline Good & Ref & & Ref & \\
\hline
\end{tabular}

Table 2 (continued)

${ }^{*} p \leq 0.05 ;{ }^{* *} p \leq 0.01 ;{ }^{* * *} p \leq 0.00$

with 2 and 19\% with 3 or more. Only $2 \%$ agreed with all 7 statements.

Unadjusted and adjusted odds ratios of medium/high internalized stigma are presented in Table 2 . Women who reported that their last pregnancy was unintended were 1.7 times more likely to have higher levels of internalized stigma compared to those who reported that their last pregnancy was intended (OR 1.7; 95\% CI 1.3-2.4). When adjusting for experience of discrimination, experience of violence or abuse and other respondent characteristics, the effect of unintended pregnancy reduced slightly to 1.6 but remained statistically significant (AOR 1.6; 95\% CI 1.2-2.3).

In the univariate models, women who experienced HIV-related discrimination and those who experienced violence were significantly more likely to have stronger feelings of internalized stigma (discrimination: OR 2.0; 95\% CI 1.5-2.8; violence: OR 1.6; 5\% CI 1.2-2.2). In the multivariate model while the association of experienced HIV-related discrimination and medium/high levels of internalized stigma remained significant (AOR: 1.8; 95\% CI 1.3-2.6), the association between violence and internalized stigma was no longer significant.

Sub-county was the only other variable that showed a significant association with internalized stigma. Those who lived in Samia sub-county were nearly 2 times more likely (AOR 1.9; 95\% CI 1.3-2.7) to have greater levels internalized stigma compared to women living in Butula sub-county. Covariates that were significant in the univariate model but were no longer significant in the multivariate model include marital status and self-reported health status.

\section{Discussion}

This study showed that internalized stigma, experience of HIV-related discrimination and unintended pregnancy remain critical issues among WLHIV in south western Kenya. Just under half of the women agreed with at least one internalized stigma statement (47\%) and 19\% of women agreed with 3-7 statements (medium/high internalized stigma). Over half (56\%) of the WLHIV surveyed said that their last pregnancy was not wanted at the time, consistent with other studies conducted in Kenya [6, 8 , 13]. Many women also reported experiencing HIVrelated discrimination (52\%), and violence or abuse (41\%) in the past 12 months. The experience of HIV-related discrimination within the past 12 months of the interview, and to some extent the experience of violence and abuse, also increased the likelihood of experiencing internalized 
stigma. While the effect of HIV-related stigma on healthseeking behaviors has been well documented [26-28], this study is one of the first to highlight the association between unintended pregnancy and internalized stigma.

WLHIV in this study were more likely to experience HIV-related internalized stigma if their last pregnancy was unintended compared to those whose last pregnancy was intended, despite experiences of discrimination or violence/abuse. Associations between internalized stigma and fertility intentions have been previously documented [42, 43]. Higher internalized stigma has been associated with higher fertility intentions in Uganda, possibly due to a desire to conform with normative behavior around childbirth, except in areas where childbearing among people living with HIV is stigmatized [42]. Similarly, personal and social pressure to be "normal" have led to people living with HIV to have children despite not wanting additional children [42]. Results from this and previous studies suggest that other factors in a woman's life, beyond being HIV-positive, influence her perceptions and feelings towards herself. There appears to be a strong link between internalized stigma and a women's fertility intentions, but the relationship may not be linear or casual. While the association between unintended pregnancy and internalized stigma remained significant when adjusting for experience of HIV-related discrimination and violence, further research is needed on whether experience of HIV-related discrimination or experience of violence mediates the association between unintended pregnancy and internalized stigma.

The timing of when a WLHIV learns of her seroconversion status (before versus during pregnancy) may influence whether a pregnancy is reported as wanted at the time or not [21] and her experience of internalized stigma [44]. While this study was unable to look at the timing of the pregnancy vis-a-vie HIV acquisition, research has shown that some WHLIV want to avoid pregnancy due to concerns of their children's wellbeing $[7,8,11]$ and therefore having an unintended pregnancy may manifested into internalized stigma, even years after pregnancy. Experiences of secondary stigma towards children have been documented and may also influences responses around pregnancy intentions [41].

More than half (57\%) of respondents who reported that their last pregnancy was unintended were not using FP at the time of the pregnancy, similar to results from previous studies [4]. As many WLHIV prefer health facilities that provide specialized services for people living with HIV $[8,29,30]$, these results suggest that WLHIV may not have the adequate access to reproductive health and FP information and services, including FP counseling for themselves and their partners, that meets their needs. A study in Kenya also found that women who were diagnosed with HIV during pregnancy and were connected to HIV care experienced lower levels of internalized stigma and depression [45]. Expanding accesses to integrated HIV/FP services or equipping health workers to provide unbiased reproductive health and FP services to WLHIV may help reduce barriers to contraception, help WLHIV plan for their families, and potentially reduce negative feelings towards themselves. In addition, some studies have found that women and couples desire to have additional children as they begin to feel healthier with ART $[10,12]$. Therefore as WLHIV's fertility intentions change over time, so do their contraceptive needs and healthcare providers should continue to regularly check in with WLHIV about those needs.

The measures of discrimination assessed in this study included a wide range of experiences and were found to be positively associated with internalized stigma. The results suggest that when a WLHIV experiences discrimination in different spheres of life, strong negative attitudes about herself can manifest. This is consistent with a study in Dominican Republic [46] that found WLHIV who reported depression and HIV-related stigma were more likely to report internalized stigma, and a study in the US which found that experienced stigma in the community had an indirect effect, through internalized stigma, on outcomes such as medication adherence and self-esteem [47], suggesting a correlation between the different levels of HIV-related stigma and discrimination. WLHIV in Samia were also more likely to experience medium/high internalized stigma compared to those in Butula. This could be because respondents from Samia sub-county are overrepresented in the analytical sample or because of other HIV projects that were being implemented in Butula sub-county at the time of the study.

While effective programs for reducing HIV-related stigma and discrimination have been well documented [48], less is known about strategies for reducing internalized stigma specifically [49]. Programs that have shown reductions in internalized stigma have addressed broader aspects in the lives of people living with HIV, such as social empowerment and economic strengthening, rather than internalized stigma directly [49]. As this study found a significant association between unintended pregnancy and internalized stigma, further research is needed to understand how reproductive health and FP programs that assist WHLIV with planning and spacing their pregnancies can also reduce internalized stigma for WLHIV. Since some WLHIV report concerns over the care and financial support of their families as reasons for not wanting additional children $[7,8,11]$, income generation and empowerment activities targeted towards WHLIV could also be explored as ways to reduce feelings of internalized stigma in the event of an unintended pregnancy. 
Health systems interventions (e.g. reducing stockouts of and expanding access to ARTs) have also been shown to reduce internalized stigma [48]. Similar health systems interventions that aim to strengthen reproductive health and FP services and increase reproductive health and FP messaging through community health workers and existing support groups may also be beneficial in reducing internalized stigma among WLHIV.

\section{Limitations}

Several limitations need to be considered in this study. One limitation to this analysis that should be considered is that respondents were not asked about when they learned of their seroconversion and therefore it is not known whether a woman's last pregnancy occurred before or after she knew her HIV status. This can lead to changes in the intendedness of the pregnancy based on when she learned of her HIV status since pregnancy intention was asked at the time of the pregnancy but reported at the time of the interview, which is when she knows her HIV status. As pregnancy intention is asked at time of the interview, another limitation is that a woman may report her last pregnancy differently at the time of the interview (which is later) than at the time of the pregnancy. Other factors in a woman's life, unrelated to HIV-status, such as martial or financial status, may influence this response at the time of the interview. Because the data were cross-sectional and unintended pregnancy was collected retrospectively, it is difficult to establish the directionality of the association and causality. Another limitation is that women enrolled in this study were all members of support groups for people living with HIV and the study did not interview WLHIV who did not belong to support groups. As result, study results pertain to a select group of women who are already motivated to seek services, and in theory should therefore have less internalized stigma.

\section{Conclusions}

This study demonstrated that unintended pregnancy is associated with internalized stigma. FP and HIV programs, and others such as maternal and antenatal care services, should continue to explore how to best support WLHIV's access to comprehensive reproductive health and FP services and continue to address HIV stigma and discrimination so that people living with HIV can access health services without fear of social or family rejection. While these results provide valuable insight for FP and HIV programming, additional research is needed on how unintended pregnancy and HIV-related discrimination influence internalized stigma among all WLHIV and whether participation in support groups mitigates experiencing internalized stigma.

\section{Abbreviations}

ART: Antiretroviral therapy; ARV: Antiretroviral; FP: Family planning; $\mathrm{RH}$ : Reproductive health; WLHIV: Women living with HIV.

\section{Supplementary Information}

The online version contains supplementary material available at https:/doi. org/10.1186/s12905-021-01224-5.

Additional file 1. The role of unintended pregnancy in internalized stigmaamong women living with HIV in Kenya.

\section{Acknowledgements}

The study was conducted in collaboration with the Busia county health management team, Samia and Butula sub-county management teams, and the Kenyan Ministry of Health. The following officials are specifically thanked for having facilitated and coordinated the implementation of project activities in Busia County: Dr. Melsa Lutomia (Busia County Director of Health); Dr. Patroba Lukale (Samia Sub-county Medical Officer of Health), Ms. Winfred Mulamba (Samia Sub-county Public Health Officer), Mr. Emmanuel Luvai (County Community Strategy Focal Person), Mr. Dennis Wanjala (Samia Sub-county Reproductive Health Coordinator), and Mr. Wilberforce Were (Butula Sub-county Reproductive Health Coordinator). We thank Michelle Hindin and Elizabeth Tobey at the Population Council who reviewed and provided editorial support for this manuscript. The successful completion of these studies would not have been possible without the dedication of the research team and data collectors. We further acknowledge all the women who volunteered their time and provided valuable information during this study.

\section{Authors' contributions}

SCD managed the overall preparation of the manuscript, including drafting the manuscript and conducting the analysis, and contributed to the manuscript's conceptualization. AJ conceptualized the manuscript, conducted preliminary analysis, and reviewed and provided comments. WL was the principal investigator for the overall study and reviewed and provided comments to the manuscript. CW contributed to the conceptualization of the overall study and reviewed and provided comments. All authors read and approved the final manuscript.

\section{Funding}

The Evidence Project is made possible by the generous support of the American people through the United States Agency for International Development (USAID) under the terms of cooperative agreement no. AID-OAA-A-13-00087. USAID funded the collection, analysis and interpretation of the data for this manuscript. The contents of this manuscript are the sole responsibility of the Evidence Project and Population Council and do not necessarily reflect the views of USAID or the United States Government.

\section{Availability of data and materials}

The dataset analyzed during the current study will be available at the USAID Development Data Library in 2021, https://www.usaid.gov/data. They will also be available from the corresponding author on reasonable request.

\section{Ethics approval and consent to participate}

The study protocol received IRB approvals from the Population Council (Protocol 702) and the Kenyatta National Hospital/University of Nairobi Ethics Research Committee (Protocol P573/08/2015). All methods were performed in accordance with the relevant guidelines and regulations stated in the approved protocol. Informed consent was received from all study participants before the start of the interviews.

\section{Consent for publication}

Not applicable. 


\section{Competing interests}

The authors declare that they have no competing interests.

\section{Author details}

1 Population Council, 4301 Connecticut Ave NW \# 280, Washington, DC 20008, USA. ${ }^{2}$ Population Council, Nairobi, Kenya.

Received: 5 November 2020 Accepted: 9 February 2021

Published online: 17 March 2021

\section{References}

1. UNAIDS, 2018. AIDS info. https://aidsinfo.unaids.org/

2. UNAIDS, 2019. Global AIDS Monitoring 2019: Indicators for monitoring the 2016 Political Declaration on Ending AIDS. https://www.unaids.org/ sites/default/files/media_asset/global-aids-monitoring_en.pdf

3. Centers for Disease Control and Prevention (CDC). Achievements in public health. Reduction in perinatal transmission of HIV infection-United States, 1985-2005. MMWR Morb Mortal Wkly Rep 2006;55(21):592-7.

4. UNAIDS, 2019. Miles To Go: Closing Gaps Breaking Barriers Righting Injustices. Global AIDS Update. https://www.unaids.org/sites/default/ files/media_asset/miles-to-go_en.pdf

5. World Health Organization. 2016. Consolidated guidelines on the use of antiretroviral drugs for treating and preventing HIV infection: recommendations for a public health approach, 2nd ed.

6. Kimani J, Warren C, Abuya T, Mutemwa R. Family planning use and fertility desires among women living with HIV in Kenya. BMC Public Health. 2015:15:909.

7. Anand A, Shiraishi RW, Bunnell RE, Jacobs K, Solehdin N, Abdul-Quader AS, et al. Knowledge of HIV status, sexual risk behaviors and contraceptive need among people living with HIV in Kenya and Malawi. AIDS. 2009;23(12):1565-73.

8. Harrington EK, Newmann SJ, Onono M, Schwartz KD, Bukusi EA, Cohen $C R$, et al. Fertility intentions and interest in integrated family planning services among women living with HIV in Nyanza Province, Kenya: a qualitative study. Infect Diseases Obstetr Gynecol. 2012;2012:809682.

9. Hoffman IF, Martinson FEA, Powers KA, Chilongozi DA, Msiska ED, Kachipapa El, et al. The year-long effect of HIV-positive test results on pregnancy intentions, contraceptive use, and pregnancy incidence among Malawian women. J Acquir Immune Defic Syndr. 2008:47(4):477-83.

10. Sarnquist CC, Rahangdale L, Maldonado Y. Reproductive health and family planning needs among HIV-infected women in Sub-Saharan Africa. Curr HIV Res. 2013;11(2):160-8.

11. Craft SM, Delaney RO, Bautista DT, Serovich JM. Pregnancy decisions among women with HIV. AIDS Behav. 2007;11(6):927-35.

12. King R, Khana K, Nakayiwa S, Katuntu D, Homsy J, Lindkvist P, et al. 'Pregnancy comes accidentally - like it did with me": reproductive decisions among women on ART and their partners in rural Uganda.'BMC Public Health. 2011;11(1):530.

13. Akelo V, Girde S, Borkowf C, Angira F, Achola K, Lando R, et al. Attitudes toward family planning among HIV-positive pregnant women enrolled in a prevention of mother-to-child transmission study in Kisumu, Kenya. PLoS ONE. 2013;8(8):e66593.

14. Kenya National Bureau of Statistics, Ministry of Health/Kenya, National AIDS Control Council/Kenya, Kenya Medical Research Institute, and National Council for Population and Development/Kenya. 2015. Kenya Demographic and Health Survey 2014. Rockville, MD, USA. http://dhspr ogram.com/pubs/pdf/FR308/FR308.pdf.

15. Adeniyi OV, Ajayi Al, Moyaki MG, Ter Goon D, Avramovic G, Lambert J. High rate of unplanned pregnancy in the context of integrated family planning and HIV care services in South Africa. BMC Health Serv. 2018:18(1):140

16. Brittain K, Philips T, Zerbe A, Abrams E, Mer L. Long-term effects of unintended pregnancy on antiretroviral therapy outcomes among South African women living with HIV. AIDS. 2019;33(5):885-93.

17. Crankshaw TL, Voce A, King RL, Giddy J, Sheon NM, Butler LM. Double disclosure bind: complexities of communicating an HIV diagnosis in the context of unintended pregnancy in Durban. South Africa AIDS Behav. 2014;18(1):53-9.
18. Salters K, Loutfy M, de Pokomandy A, Money D, Pick N, Wang L, et al. Pregnancy incidence and intention after HIV diagnosis among women living with HIV in Canada. PLoS ONE. 2017;12(7):e0180524.

19. Sutton MY, Patel R, Frazier EL. Unplanned pregnancies among HIVinfected women in care-United States. J Acquir Immune Defic Syndr. 2014;65(3):350-8.

20. Mersha AG, Erku DA, Belachew SA, Ayele AA, Gebresillassie BM, Abegaz TM. Contraceptive use among HIV-positive and negative women: implication to end unintended pregnancy. Contracept Reprodu Med. 2019;4:3-3.

21. Iyun V, Brittain K, Phillips TK, le Roux S, Mclntyre JA, Zerbe A, et al. Prevalence and determinants of unplanned pregnancy in HIV-positive and HIV-negative pregnant women in Cape Town, South Africa: a cross-sectional study. BMJ Open. 2018;8(4):e019979.

22. Bankole A, Keogh S, Odunayo A, Dzekedzeke K, Awolude O, Adewole I. Differences in unintended pregnancy, contraceptive use and abortion by HIV status among women in Nigeria and Zambia. Int Perspect Sex Reprod Health. 2014;40(1):28-38.

23. National AIDS and STI Control Programme (NASCOP), Kenya. Kenya AIDS Indicator Survey 2012: Final Report. Nairobi, NASCOP; 2014.

24. Center for Disease Control. Dealing with Stigma and Discrimination. Accessed on August 6, 2019. https://www.cdc.gov/hiv/basics/livin gwithhiv/stigma-discrimination.html\#: :text=HIV\%2Drelated\%20sti gma\%20refer\%20to,youth\%2C\%20and\%20mentally\%20ill\%20people.

25. Center for Disease Control. 2018. Internalized HIV-related Stigma. Medical Monitoring Report. https://www.cdc.gov/hiv/pdf/statistics/mmp/ cdc-hiv-internalized-stigma.pdf

26. Turan JM, Bukusi EA, Onono M, Holzemer WL, Miller S, Cohen CR. HIV/ AIDS stigma and refusal of HIV testing among pregnant women in rura Kenya: results from the MAMAS Study. AIDS Behav. 2011;15(6):1111-20.

27. Obare $F$, van der Kwaak A, Birungi $H$. Factors associated with unintended pregnancy, poor birth outcomes and post-partum contraceptive use among HIV-positive female adolescents in Kenya. BMC Women's Health. 2012;12(1):34.

28. Katz IT, Ryu AE, Onuegbu AG, Psaros C, Weiser S, Bangsberg DR, et al. Impact of HIV-related stigma on treatment adherence: systematic review and meta-synthesis. J Int AIDS Soc. 2013;16(3 Suppl 2):18640-18640.

29. Turan JM, Miller S, Bukusi EA, Sande J, Cohen CR. HIV/AIDS and maternity care in Kenya: how fears of stigma and discrimination affect uptake and provision of labor and delivery services. AIDS Care. 2008;20(8):938-45.

30. Colombini M, Mutemwa R, Kivunaga J, Stackpool Moore L, Mayhew S. Experiences of stigma among women living with HIV attending sexual and reproductive health services in Kenya: a qualitative study. BMC Health Serv Res. 2014;14:412-412.

31. Turan JM, Nyblade L. HIV-related stigma as a barrier to achievement of global PMTCT and maternal health goals: a review of the evidence. AIDS Behav. 2013;17(7):2528-39.

32. Rice WS, Crockett KB, Mugavero MJ, Raper JL, Atkins GC, Turan B. Association between internalized HIV-related stigma and HIV care visit adherence. J Acquired Immune Defic Syndr. 2017;76(5):482-7.

33. Turan B, Rice WS, Crockett KB, Johnson M, Neilands TB, Ross SN, Kempf MC, Konkle-Parker D, Wingood G, Tien PC, Cohen M, Wilson TE, Logie CH, Sosanya O, Plankey M, Golub E, Adimora AA, Parish C, Weiser SD, Turan JM. Longitudinal association between internalized HIV stigma and antiretroviral therapy adherence for women living with HIV: the mediating role of depression. AIDS. 2019;33(3):571-6.

34. Turan B, Smith W, Cohen MH, Wilson TE, Adimora AA, Merenstein D, Adedimeji A, Wentz EL, Foster AG, Metsch L, Tien PC, Weiser SD, Turan JM. Mechanisms for the negative effects of internalized HIV-related stigma on antiretroviral therapy adherence in women: the mediating roles of social isolation and depression. J Acquir Immune Defic Syndr. 2016;72(2):198-205.

35. Logie CH, Lacombe-Duncan A, Wang Y, Kaida A, Conway T, Webster K, de Pokomandy A, et al. Pathways from HIV-related stigma to antiretroviral therapy measures in the HIV care cascade for women living with HIV in Canada. J Acquir Immune Defic Syndr. 2018;77(2):144-53.

36. Brittain K, Mellins CA, Phillips T, Zerbe A, Abrams EJ, Myer L, et al. Social support, stigma and antenatal depression among HIV-infected pregnant women in South Africa. AIDS Behav. 2017;21(1):274-82.

37. Kenya National Bureau of Statistics. 2019 Kenya Population and Housing Census. Kenya Data Portal. https://kenya.opendataforafrica.org/msdpn 
bc/2019-kenya-population-and-housing-census-population-by-countyand-sub-county?county $=1003270$-busia

38. Liambila W, Chace Dwyer S, Mdawida B, Warren C, Lutomia M, Koech J, et al. Strengthening the Integration of Family Planning and HIV Services at the Community Level in Kenya. Research Report. 2018 Washington, DC \& Nairobi, Kenya: Population Council, The Evidence Project.

39. National AIDS and STI Control Programme (NASCOP), Preliminary KENPHIA 2018 Report. Nairobi: NASCOP; 2020

40. National AIDS Control Council. 2018. The Kenya HIV Estimates Dashboard. https://nacc.or.ke/2018-hiv-estimates/

41. Berger BE, Ferrans CE, Lashley FR. Measuring stigma in people with HIV: psychometric assessment of the HIV stigma scale. Res Nurs Health. 2001;24(6):518-29.

42. Wagner G, Linnemayr S, Kityo C, Mugyenyi P. Factors associated with intention to conceive and its communication to providers among HIV clients in Uganda. Maternal Child Health J. 2012;16:510-8.

43. Nattabi B, Li Jianghong, Thompson SC, Orach CG, Earnest J. Between a rock and a hard place: stigma and the desire to have children among people living with HIV in northern Uganda. J Int AIDS Soc. 2012:15(2):17421.

44. Cichowitz C, Mazuguni F, Minja L, Njau P, Antelman G, Ngocho J, Knettel BA, Watt MH, Mmbaga BT. Vulnerable at each step in the PMTCT care cascade: high loss to follow up during pregnancy and the postpartum period in Tanzania. AIDS Behav. 2019;23(7):1824-32.
45. Turan B, Stringer KL, Onono M, et al. 2014 Linkage to HIV care, postpartum depression, and HIV-related stigma in newly diagnosed pregnant women living with HIV in Kenya: a longitudinal observational study. BMC Pregnancy Childbirth. 2014;14:400.

46. Rael CT, Hampanda K. Understanding internalized HIV/AIDS-related stigmas in the Dominican Republic: a short report. AIDS Care. 2016;28(3):319-24.

47. Kay ES, Rice WS, Crockett KB, Atkins GC, Batey DS, Turan B. Experienced HIV-related stigma in health care and community settings: mediated associations with psychosocial and health outcomes. J Acquir Immune Defic Syndr. 2018;77(3):257-63.

48. Pantelic M, Sprague L, Stangl AL. It's not "all in your head": critical knowledge gaps on internalized HIV stigma and a call for integrating social and structural conceptualizations. BMC Infect Dis. 2019;19(1):210.

49. Pantelic M, Steinert Jl, Park J, Mellors S, Murau F. 'Management of a spoiled identity': systematic review of interventions to address selfstigma among people living with and affected by HIV. BMJ Global Health. 2019;4:e001285. https://doi.org/10.1136/bmjgh-2018-001285.

\section{Publisher's Note}

Springer Nature remains neutral with regard to jurisdictional claims in published maps and institutional affiliations.
Ready to submit your research? Choose BMC and benefit from:

- fast, convenient online submission

- thorough peer review by experienced researchers in your field

- rapid publication on acceptance

- support for research data, including large and complex data types

- gold Open Access which fosters wider collaboration and increased citations

- maximum visibility for your research: over $100 \mathrm{M}$ website views per year

At BMC, research is always in progress.

Learn more biomedcentral.com/submissions 